\title{
Feasibility and tolerability of self-insertion of a transnasal tube for esophagogastroduodenoscopy: a pilot study
}

\author{
Before the intervention \\ - During the consult, it is suggested to the patient that he/she \\ participate in the self-insertion procedure. \\ - The entire process is explained. \\ - The patient gives consent for the self-insertion procedure, in \\ particular agreeing to look at his/her anatomy on a dedicated \\ screen throughout the procedure. \\ Perioperative period \\ - The room and the patient are prepared for the procedure, \\ and the patient is seated or half-seated facing a screen. \\ - Local anesthesia (nose and mouth) is given. \\ - The entire procedure is reviewed with the patient \\ (anatomy, difficult steps of the procedure). \\ - The physician builds the patient's confidence. \\ - The patient agrees to follow all instructions. \\ - The patient self-inserts the tube. \\ - The physician explains the anatomy that the patient \\ sees on the screen. \\ - The physician coaches the patient throughout the procedure, \\ providing instructions (e.g., "go forward," "stop," "slowly") \\ and support. \\ After the intervention \\ - The patient is given the results of the examination. \\ - The tolerability is assessed, and the patient's feelings \\ about the self-insertion procedure are discussed.
}

Recently, Quilliot et al. reported, for the first time, that self-insertion of a nasogastric tube may be efficacious and well tolerated in patients receiving enteral nutrition [1]. A similar process was used to increase the tolerability of diagnostic esophagogastroduodenoscopy (EGD) and the confidence of patients undergoing this procedure.

A single senior physician with a high level of experience in endoscopy suggested to 10 outpatients referred for diagnostic EGD that they self-insert a transnasal endoscope after the administration of topical anesthesia ( Fig. 1). The patients were counseled as follows: "This new technique enables you to take control of the endoscopic procedure completely because you will introduce the tube yourself after you have received local anesthesia. You will view the process on a screen, and I will coach you on how to insert and guide the tube." Before the examination, patients were given a description of the anatomy involved and the procedure. All patients gave informed consent. After the procedure, they were asked if they would agree to use this technique again.
Fig. 1 Essential steps of the procedure for self-insertion of a nasogastric tube by a patient undergoing esophagogastroduodenoscopy.

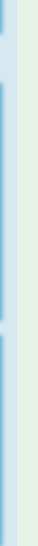

patient completed the procedure but with poor tolerance, probably because she did not follow the examination on the screen and, with eyes closed, only listened to the instructions given by the physician. The last patient refused at first to use the self-insertion technique because of previous aesthetic nose surgery. When the classic endoscopic procedure had to be stopped because of nausea and vomiting, the new technique was again suggested; she agreed this time, and the procedure was a complete success. All patients were extremely satisfied and agreed to self-insert the tube again if another examination were to become necessary.

Self-insertion of a transnasal tube for diagnostic EGD is feasible if patients can accept the idea of viewing their own anatomy. The procedure is extremely well tolerated and may decrease patients' pain and discomfort.

\section{Endoscopy_UCTN_Code_TTT_1AO_2AN}

Competing interests: Dr. Senesse has received honoraria or speaker's fees from Baxter, Fresenius Kabi, Nestlé HomeCare, Lactalis, and Nutricia; has served in a consulting or advisory role for Aguettant, Baxter, Fresenius Kabi, and Nestlé International; has received research funding from Ipsen and Nestlé International; and has received remuneration for travel and accommodations from Aguettant, AVEO Pharmaceuticals, Baxter France, Fresenius Kabi, and Nestlé HomeCare. The other authors have no conflicts of interest to disclose.

\section{Pierre Senesse ${ }^{1,2,3}$, Geneviève \\ Georges $^{1}$, Sylvie Séville ${ }^{1}$, Nicolas Flori ${ }^{1}$, Anne Stoebner ${ }^{4}$, Eric Assenat ${ }^{1}$, Hélène de Forges ${ }^{5}$}

${ }^{1}$ Department of Clinical Nutrition and Gastroenterology, Institut régional du Cancer de Montpellier (ICM), Montpellier, France

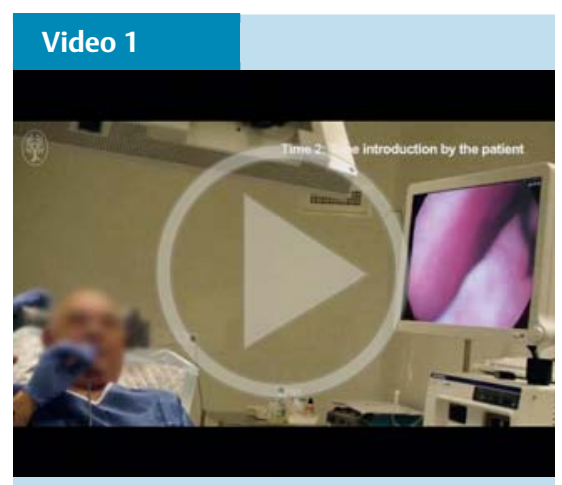

Self-insertion of a nasogastric tube by a patient undergoing esophagogastroduodenoscopy.
2 SIRIC Montpellier Cancer, Institut régional du Cancer de Montpellier (ICM),

Montpellier, France

${ }^{3}$ Epsylon Laboratory, EA 4556 Dynamics of Human Abilities and Health Behaviors, University of Montpellier, France

${ }^{4}$ Patient Education Centre, Department of Supportive Care , Institut régional du Cancer de Montpellier (ICM), Montpellier, France

${ }^{5}$ Clinical Research Department, Institut régional du Cancer de Montpellier (ICM), Montpellier, France 


\section{Acknowledgments}

We thank all the patients who participated in the study, especially the patient who agreed to allow us to make a video recording of his procedure and show it. We also thank, for their technical work on the video, Margaux Colliot of the Communication Department, Institut régional du Cancer de Montpellier (ICM), and the members of the K'Noé Society, especially Aurore Jouval and Bertrand Goichot.

\section{Reference}

1 Quilliot D, Zallot C, Malgras A et al. Self-insertion of a nasogastric tube for home enteral nutrition: a pilot study. JPEN J Parenter Enteral Nutr 2014; 38: 895-900

Bibliography

Dol http://dx.doi.org/

10.1055/s-0034-1392795

Endoscopy 2015; 47: E475-E476

(c) Georg Thieme Verlag KG

Stuttgart · New York

ISSN 0013-726X

\section{Corresponding author}

\section{Pierre Senesse, MD, PhD}

Department of Clinical Nutrition and Gastroenterology

Institut régional du Cancer de Montpellier (ICM) Val d'Aurelle

208 avenue des Apothicaires

34298 Montpellier

France

Fax: +33-04-67-61-37-39

Pierre.Senesse@icm.unicancer.fr 